\title{
Hospitationspraktika als Lerngelegenheit? Zum Beitrag von Praktika zur Veränderung berufsbezogener Selbstkonzepte und der Berufswahlsicherheit bei Lehramtsstudierenden
}

\author{
Tabea Kauper (D)
}

Angenommen: 18. Oktober 2018 / Online publiziert: 1. November 2018

(C) Der/die Autor(en) 2018

Zusammenfassung Berufsbezogene Selbstkonzepte und die Berufswahlsicherheit haben eine große Bedeutung für die Professionsentwicklung angehender Lehrkräfte. Wie sich berufsbezogene Selbstkonzepte und die Berufswahlsicherheit durch Hospitationspraktika verändern können, ist jedoch eine bislang offene Forschungsfrage. Ziel der vorliegenden Studie ist es daher, erstens Veränderung in den berufsbezogenen Selbstkonzepten und der Berufswahlsicherheit durch das Hospitationspraktikum zu untersuchen und zweitens, den Einfluss der intendierten Praktikumsaufgaben auf diese Veränderung hin zu analysieren.

Zur Beantwortung der ersten Frage wurden zwei Studierendengruppen vergleichend untersucht. Eine Gruppe absolvierte ein Hospitationspraktikum, die zweite Gruppe absolvierte in diesem Zeitraum kein Praktikum. Untersucht wurde, ob sich die beiden Gruppen hinsichtlich ihres Selbstkonzepts und der Berufswahlsicherheit vor und nach dem Praktikum unterscheiden. Anschließend wurde anhand der Gruppe mit absolviertem Praktikum die Veränderung des Selbstkonzepts sowie der Berufswahlsicherheit durch die intendierten Aufgaben des Praktikums und unter Berücksichtigung der Betreuung und der Persönlichkeit näher betrachtet. Den Analysen liegt eine drei Erhebungswellen umfassende Längsschnittstichprobe an 296 Studierenden (Praktikumsgruppe) bzw. 527 Studierenden (Vergleichsgruppe) aller Fächerkombinationen aus neun Hochschulen zugrunde. Die Selbstkonzepte und die Berufswahlsicherheit wurden im Sinne eines Prä-Post-Designs vor und nach dem Praktikum erhoben.

\footnotetext{
Zusatzmaterial online Zusätzliche Informationen sind in der Online-Version dieses Artikels (https://
} doi.org/10.1007/s35834-018-0225-8) enthalten. 
Der Gruppenvergleich zeigt, dass sich die Selbstkonzepte und die Berufswahlsicherheit nicht vor, aber nach dem Praktikum zwischen den Gruppen unterscheiden. Analysen zur Entwicklung im Praktikum legen Einflüsse sowohl der intendierten Praktikumsaufgaben, als auch der Betreuung sowie hohe negative Einflüsse des Persönlichkeitsmerkmals Neurotizismus offen. Zusammengefasst zeigen die Ergebnisse, dass die Lerngelegenheit Hospitationspraktikum einen Beitrag zur Veränderung der berufsbezogenen Selbstkonzepte, aber vor allem für die Berufswahlsicherheit leistet.

Schlüsselwörter Hospitationspraktikum · Orientierungspraktikum · Berufsbezogenes Selbstkonzept · Sicherheit der Berufswahl

\title{
Initial teacher training as learning opportunity? The impact of teacher trainings on the development of professional self-concept and certainty of career choices
}

\begin{abstract}
The present study examines the effectiveness of initial teacher training on the development of professional self-concept and certainty of career choices of teacher students in Germany. The focus of the study is on the one hand whether these teacher trainings affect the career orientation as intended in their curriculum investigated exemplarily by the development of self-concept and certainty of career choices and on the other hand predict the intended learning tasks the development of both.

In order to answer the first question, two groups of students were compared. One group completed the initial teacher training; the second group did not complete this training during this period of their teacher training. The study examined whether the groups differ in terms of their self-concept and certainty of career choice before and after the internship. Furthermore, the development of self-concept and certainty of career choices were examined through the tasks of the teacher training under the condition of quality of support and personality traits on the basis of the group with completed training. Longitudinal data with three measurement points were obtained of a sample of 296 students undergoing teacher training resp. 527 teacher students without conducting a teacher training in nine universities.

Group comparisons show that the self-concept and certainty of career choice do not differ before but after the teacher training between both groups. Further analyses of the development during teacher training reveal effects of the intended learning tasks on self-concept and mentoring as well as negative influences of neuroticism as a trait of students' personality. In summary, results show that the learning opportunities of initial teacher trainings provide a contribution to the professional development of teacher students.
\end{abstract}

Keywords Practical teacher trainings - Field experiences - Professional selfconcept $\cdot$ Certainty of career choice 


\section{Einleitung}

Die Bedeutung der Praxisphasen als Lerngelegenheit in der ersten Phase der Lehramtsausbildung wurde durch den Quedlinburger Beschluss der KMK (2005) bestätigt und kann aktuell durch die bundesweite Tendenz der zeitlichen Ausdehnung zu Praxissemestern bei gleichzeitig verbesserter curricularer Einbindung und Lernbegleitung in Deutschland beobachtet werden (Weyland 2012). Praxissemester sind im Master- bzw. Hauptstudium angesiedelt, für das Grund- oder Bachelorstudium sind dagegen zeitlich kurze Praktika nach wie vor ein fester Bestandteil des Curriculums. Diese ersten Schulpraktika, mit denen die Studierenden konfrontiert sind, dauern zwischen 10 und 40 Tagen und sollen vorrangig der Orientierung der Studierenden dienen (Gröschner et al. 2015). Ihnen kommt die Funktion einer ,Schnittstelle zwischen universitärer Ausbildung und ersten Erfahrungen Studierender im Praxisfeld Schule“ zu (Lazarides und Mohr 2015, S. 381). Diese Praktika werden je nach Hochschule bspw. als Kennenlern-, Berufsfelderkundungs-, Orientierungs-, Eingangs- oder Hospitationspraktikum benannt (vgl. Topsch 2004). In der vorliegenden Studie wird die Bezeichnung „Hospitationspraktikum“ verwendet.

Studierende schätzen Praxisphasen als Lerngelegenheit und erleben durch Praktika einen Kompetenzzuwachs, den sie als berufstätige Lehrer jedoch deutlich kritischer einschätzen, als unmittelbar nach dem Praktikum (Hascher 2006). Empirische Ergebnisse zeigen Zuwächse in den selbsteingeschätzten Kompetenzen bspw. zum Unterrichten (Gröschner et al. 2013) oder Innovieren (Rothland und Straub 2018; Gröschner et al. 2013). Es finden sich aber auch eine Reihe unerwünschter Effekte, die die Vermutung nahelegen, dass dem wahrgenommenen Kompetenzzugewinn keine Lern-, sondern Sozialisationsprozesse zugrunde liegen, bei denen der Mentor/die Mentorin unreflektiert als Vorbild dient und imitiert wird (Hascher 2012; Rothland und Boecker 2014). Trotz einer deutlich verstärkten Forschung zu schulischen Praxisphasen in den letzten Jahren gibt es nur wenig empirische Evidenz für deren Wirksamkeit (z. B. Hascher 2012; Hascher und de Zordo 2015; Rothland und Boecker 2014, 2015).

Ziel der vorliegenden Studie ist es, die Wirkung des Hospitationspraktikums aus hochschulübergreifender Perspektive auf das berufsbezogene Selbstkonzept und die subjektiv wahrgenommene Berufswahlsicherheit von Lehramtsstudierenden zu untersuchen. Berufsbezogene Selbstkonzepte umfassen ihrerseits verschiedene Kompetenzbereiche, die für den späteren Beruf als wichtig angenommen werden können (Retelsdorf et al. 2014). Zudem gelten Selbstkonzepte als wichtige Prädiktoren für Leistung und beruflichen Erfolg, neben einer Vielzahl weiterer psychologischer Variablen (Marsh und Martin 2011; Möller und Marsh 2013). Über deren Veränderung im Zuge des Lehramtsstudiums ist hingegen nur wenig bekannt (vgl. Rothland und Straub 2018). Die empfundene Sicherheit der Berufswahl ist wiederum prädiktiv für die Aufrechterhaltung des Interesses an den Studieninhalten und der Studienmotivation (Rösler et al. 2013). Beide Konstrukte, sowohl die berufsbezogenen Selbstkonzepte als auch die Berufswahlsicherheit, beziehen sich unmittelbar auf die (zukünftige) Berufstätigkeit der Studierenden, indem sie entweder Berufsanforderungen oder eine gelungene Berufswahl abbilden. Dadurch stehen diese Variablen einerseits im engen Zusammenhang mit der geforderten Berufswahlüberprüfung durch Praxis- 
phasen (vgl. auch Weyland 2012) und können andererseits auch als Kriterien zur Berufsorientierung verstanden werden.

\section{Theoretischer Hintergrund}

\subsection{Aufgaben, Ziele und institutionelle Rahmenbedingungen von Hospitationspraktika}

Vorrangiges Ziel der Praxisphasen ist es, eine Verbindung zwischen „Theorie“ - im Sinne des Studiums - und „Praxis“ - im Sinne der zukünftigen Berufstätigkeit während der ersten Phase der Lehramtsausbildung zu schaffen (z. B. Bennack und Jürgens 2002). Nach Terhart (2000) entspricht dies einer Selbst- und Theoriereflexion auf Basis des in den Praktika erworbenen Wissens, Könnens und Problembewusstseins sowie der Überprüfung des Berufswunsches. Aus diesen Zielen lassen sich drei Hauptaufgaben ableiten, dazu gehört erstens die theoriegeleitete Beobachtung und Reflexion der Unterrichtspraxis. Diese Aufgabe dient maßgeblich der Verknüpfung von Theorie und Praxis im Sinne der Überprüfung wissenschaftlicher Theorien und Konzepte an der Schulwirklichkeit. Als zweite übergeordnete Aufgabe ist die Einübung in die Unterrichtspraxis und in die Lehrendenrolle zu sehen. Dabei soll die eigene Unterrichtspraxis reflektiert und professionelle Handlungskompetenz angebahnt werden. Eng damit einher geht die dritte Aufgabe, die der Berufswahlüberprüfung. Diese Aufgabe hat als Ziel, die Versachlichung und Reflexion der Berufsorientierung zu erreichen (z. B. Reinhoffer und Dörr 2008; Topsch 2004) und ist nach Topsch (2004) vor allem als Aufgabe des Hospitationspraktikums zu sehen (vgl. auch Arnold et al. 2011).

Die vorliegende Studie nutzt als Datengrundlage Hochschulen, bei denen Auswertungen zu den Praxisphasen anhand des intendierten Curriculums bereits vorliegen (Gröschner et al. 2015). So konnten Gröschner et al. (2015) zeigen, dass an allen 12 untersuchten Hochschulen das jeweils erste Praktikum der Professionalisierungsabsicht „Orientierung“ dient und daher als Orientierungs- bzw. Hospitationspraktikum bezeichnet werden kann. Es werden jedoch verschiedene Aspekte der Orientierung betont, das Spektrum reicht vom Kennenlernen der Schule oder des Berufsfeldes ,als Ganzes“ bis zum Kennenlernen einzelner Facetten (,Arbeitsfeld Schule als Lernund Erfahrungsraum“, ebd. S. 653), von der Berufswahlreflexion bis hin zur TheoriePraxis-Verknüpfung. Die Studierenden sollen sich im Beobachten üben, sich ihrer (zukünftigen) erzieherischen Verantwortung bewusst werden und der Rollenwechsel vom Schüler/in zum/zur Lehrer/in soll angebahnt werden.

Eine besondere Rolle für das Gelingen von Praxisphasen im Hinblick auf die Professionsentwicklung kommt der Betreuung zu. Es ist nach aktuellem Forschungsstand nicht davon auszugehen, dass Praktika ohne eine theoretische und praktische Einführung in Unterrichtsbeobachtung (Bennack und Jürgens 2002), einer universitären Betreuung (Büscher 2004; Hascher 2006; Schubarth et al. 2011) oder mentoriellen Begleitung (Hascher und Moser 1999, 2001) im Sinne erwünschter Lerneffekte gelingen können. Bach et al. (2014) konnten zeigen, dass vor allem die Qualität der Beziehung zum/zur Mentor/in im Zusammenhang mit Kompetenzentwicklung steht. 
Betreuungsvarianten wie der Schulbesuch von Hochschuldozierenden werden von Studierenden im Hospitationspraktikum als nicht nützlich eingeschätzt, Feedbacks $\mathrm{zu}$ den eigenen Unterrichtsversuchen hingegen positiv wahrgenommen (Lazarides und Mohr 2015).

Nach Schubarth et al. (2011) muss weiterhin berücksichtigt werden, dass die Wahrnehmung der Betreuung auch von Persönlichkeitsmerkmalen und praktischen Vorerfahrungen der Studierenden beeinflusst wird. Persönlichkeitsmerkmale nehmen darüber hinaus auch Einfluss auf die Kompetenzselbsteinschätzungen und die Nutzung von Lerngelegenheiten (Müller 2010).

\subsection{Berufsbezogene Selbstkonzepte und Berufswahlsicherheit als Kompetenzfacetten der Lehrkräfteprofessionalisierung}

In Anlehnung an das Modell professioneller Handlungskompetenz von Baumert und Kunter (2006, 2011) setzt sich diese aus Professionswissen, Überzeugungen und Werthaltungen, Selbstregulation und motivationalen Orientierungen zusammen. $\mathrm{Zu}$ letzteren gehören auch die selbstbezogenen Kognitionen, wie bspw. das berufsbezogene Selbstkonzept, als wichtige Facette professioneller Kompetenz (Retelsdorf et al. 2014). Im beruflichen Kontext beeinflusst das Selbstkonzept wie Personen ihre professionellen Fähigkeiten einschätzen und in ihrer beruflichen Tätigkeit konkretisieren (Scheller und Filipp 1995). Zudem gilt ein positives Selbstkonzept als hoch prädiktiv für akademische und berufliche Leistungen. Aktuelle Studien stellen das Selbstkonzept als einen der wichtigsten Prädiktoren zur Erklärung von beruflichem Erfolg heraus (vgl. Marsh und Martin 2011). Umgekehrt zeigen Studien, dass bei belasteten Lehrkräften neben weiteren Faktoren auch ungünstige Selbstkonzepte beobachtet werden können (Lipowsky 2003). Über die Veränderung berufsbezogener Selbstkonzepte ist hingegen nur sehr wenig bekannt. So finden Rothland und Straub (2018) nur sehr geringe Veränderungen in den berufsbezogenen Selbstkonzepten bei hoch ausgeprägten Selbsteinschätzungen vor einem Praxissemester, Schwippert et al. (2013) beobachten keine Veränderung im akademischen Selbstkonzept bei gleichzeitigem Vorliegen mittlerer Ausgangswerte zu Studienbeginn. Zu gegenläufigen Befunden kommen hingegen querschnittliche Vergleiche zwischen Studienanfängern und Studierenden in fortgeschrittenen Fachsemestern, letztere berichten niedrigere Selbstkonzepte als die Studienanfänger, was als Relativierung eigener Fähigkeitseinschätzungen durch die Konfrontation mit den Studienanforderungen interpretiert wird (Retelsdorf et al. 2014).

Befunde zur Berufswahlsicherheit zeigen, dass grundsätzlich von einer hohen Stabilität ausgegangen werden kann, die bei angehenden Grundschullehrkräften etwas höher ausgeprägt ist, als bei angehenden Gymnasiallehrkräften (Bauer et al. 2011). In Anlehnung an Rösler et al. (2013) kann die Berufswahlsicherheit als gelungene Berufswahl interpretiert werden, die sich wiederum positiv auf die Entwicklung studienbezogener Interessen und damit auf eine vertiefte Beschäftigung mit den Inhalten der Studienbereiche auswirkt. Hinzu kommt, dass die Berufswahlüberprüfung ein wichtiges Ziel des Hospitationspraktikums ist. Diese Überprüfung soll erreicht werden, indem die angehenden Lehrkräfte mit dem Berufsfeld aus Sicht des Lehrenden bzw. mit den Anforderungen der Praxis konfrontiert werden. Sollte 
reflektiert werden, so müsste sich das in der Berufswahlsicherheit der Studierenden wiederfinden (vgl. Rothland und Boecker 2015).

Insgesamt gibt es nur wenig Forschung, die sich dezidiert mit der Entwicklung nicht direkt wissensbezogener Kompetenzen im Hospitationspraktikum beschäftigt. Empirische Studien, die sich hingegen mit dem Wissenserwerb in Hospitationspraktika beschäftigen (bspw. König 2012; König et al. 2012), stellen neben der Absolvierung des Praktikums die Bedeutung von Unterrichtsversuchen heraus, die jedoch nicht in jedem Studienmodell curricular vorgesehen sind (Darge et al. 2012; Gröschner et al. 2015).

\section{Fragestellung}

Vor dem skizzierten Hintergrund und der o. a. Forschungsdesiderata steht die Veränderung der berufsbezogenen Selbstkonzepte am Beispiel der Selbstkonzeptbereiche Fach und Erziehung sowie der Berufswahlsicherheit durch das Hospitationspraktikum im Fokus der Studie. Um etwaige Veränderungen auf die Lerngelegenheit Hospitationspraktikum interpretieren zu können, wurden dazu zunächst zwei Studierendengruppen vergleichend untersucht. Eine Gruppe absolvierte ein Hospitationspraktikum (Praktikumsgruppe), die zweite Gruppe absolvierte in dem Zeitraum kein Praktikum (Vergleichsgruppe). Geprüft wurde, ob sich die beiden Gruppen hinsichtlich ihres Selbstkonzepts und der Berufswahlsicherheit zum Zeitpunkt vor und nach dem Praktikum unterscheiden.

Anschließend wurde anhand der Praktikumsgruppe die Veränderung der Selbstkonzeptbereiche Fach und Erziehung und der Berufswahlsicherheit näher betrachtet. Dazu wurde als zweite Forschungsfrage untersucht, ob die Einschätzung der Studierenden zu den intendierten Aufgaben des Praktikums „Erfahrungen im schulischen Kontext sammeln“, „Berufswahlreflexion“ sowie „Theorie-Praxis-Transfer“ Veränderung in den genannten Selbstkonzeptbereichen sowie der Berufswahlsicherheit prädizieren. Weiterhin wurde untersucht, welchen Einfluss die wahrgenommene Betreuungsqualität sowie die Persönlichkeit der Studierenden auf die Einschätzungen

T1

T2

T3

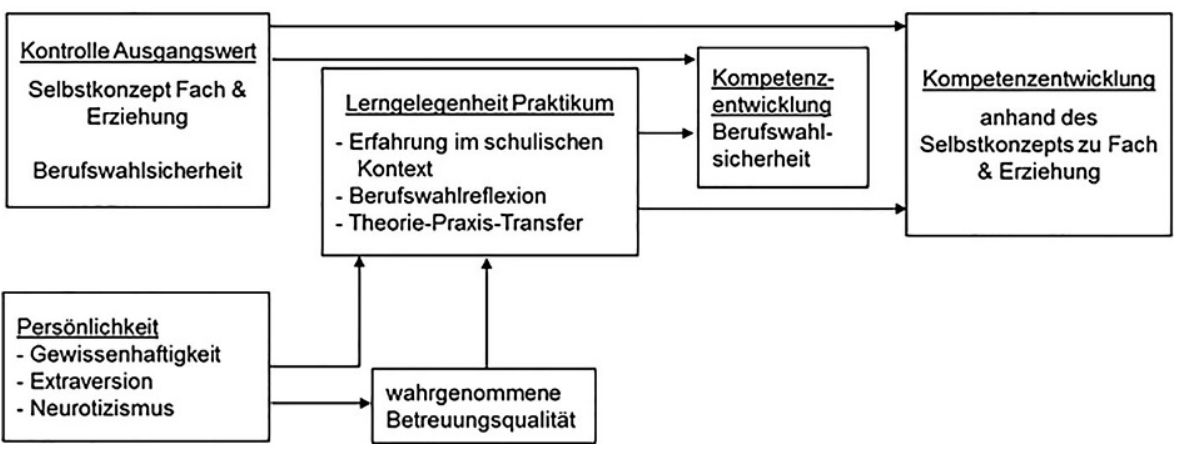

Abb. 1 Konzeptuelles Modell zur Veränderung der berufsbezogenen Selbstkonzepte Fach und Erziehung sowie der Berufswahlsicherheit durch Hospitationspraktika 
dieser Aufgaben nehmen. Es wurde außerdem geprüft, ob die Persönlichkeit darüber hinaus die Wahrnehmung der Betreuung beeinflusst. Das untersuchungsleitende Modell zur zweiten Forschungsfrage ist Abb. 1 entnehmbar.

\section{Methode}

\subsection{Stichprobe}

Der Studie liegen Daten aus drei Messzeitpunkten des Projekts „PaLea - Panel zum Lehramtsstudium“ (Bauer et al. 2010) zugrunde. Bei der vorliegenden Studie wurde eine Teilstichprobe derjenigen Studierenden verwendet, die ein Lehramtsstudium zum Wintersemester 2009/10 bzw. 2010/11 aufgenommen und im ersten Semester inkl. vorlesungsfreier Zeit ein Schulpraktikum absolvierten (Praktikumsgruppe). Zur Absicherung der Praktikumseffekte auf die abhängigen Merkmale wurde eine Vergleichsstichprobe von Lehramtsstudierenden derselben Universitäten einbezogen, die in diesem Zeitraum kein Praktikum absolvierten (Vergleichsgruppe).

Die Studierenden wurden über ihre Hochschule kontaktiert und füllten zu Beginn ihres Studiums (Wintersemester 2009/2010 bzw. 2010/11, T1) einen Fragebogen innerhalb einer Lehrveranstaltung aus. Für die anschließenden Erhebungswellen wurden die Studierenden via Email angeschrieben und zu einer Online-Erhebung eingeladen. Die weiteren hier berücksichtigten Messzeitpunkte liegen zu Beginn (T2) und zum Ende (T3) des zweiten Semesters. Der zeitliche Abstand beträgt dementsprechend zwischen den ersten beiden Messzeitpunkten ca. fünf bis sechs Monate, zwischen dem zweiten und dritten Messzeitpunkt drei Monate.

Praktikumsgruppe Die Stichprobe der Praktikumsgruppe beläuft sich auf insgesamt 296 Lehramtsstudierende an neun Hochschulen (74\% weiblich; Alter $M=21,3$ Jahre, $S D=3,7)$. An drei der 12 Hochschulen der ursprünglichen Datenbasis (s. Abschn. 2.1, Gröschner et al. 2015) wurde zu diesem frühen Zeitpunkt kein Praktikum angeboten, sie entfallen daher in den Analysen.

Die meisten Befragten $(40 \%, n=117)$ studieren in einem gymnasialen Lehramtsstudiengang, gefolgt von angehenden Grundschullehrkräften $(29 \%, n=85)$. Die Dauer des Hospitationspraktikums betrug im Mittel zwischen zwei und vier Wochen $(M=3,11, S D=1,2$, Range: $1-7)$. Es wurden insgesamt 64 Studierende aus den Analysen ausgeschlossen, die Extremwerte in der Praktikumsdauer angaben (Dauer $\geq 8$ Wochen).

Nahezu alle Studierenden gaben „hospitieren“ als hauptsächliche Tätigkeit im Praktikum an $(96,8 \%)$. Weitere $81,5 \%$ der Studierenden bereiteten mindestens gelegentlich und $12 \%$ nie eine Unterrichtsstunde vor, im Mittel wurden 3,56 ( $S D=2,77$, Range 0-12) Unterrichtsstunden gehalten. Es nahmen weiterhin $44 \%$ der Studierenden an außerschulischen Veranstaltungen teil und 15,1\% an „Elternabenden/gesprächen“.

Weiterhin wurden vier Betreuungsvarianten hinsichtlich ihres Vorhandenseins eingeschätzt. So erlebten $26 \%$ der Studierenden individuelle Betreuung an ihrer Universität, $93 \%$ individuelle Betreuung an der Praktikumsschule und $4 \%$ eine Be- 
treuung an der Schule durch Personal der Hochschule. Eine Betreuung durch vor-, nachbereitende oder begleitende Lehrveranstaltung erlebten $63 \%$ der Studierenden. Es gaben weiterhin 8 Studierende (2,9\%, aus fünf Universitäten) an, keine Betreuung erhalten zu haben.

Intraklassenkorrelationen (ICC) zur Bestimmung des Zusammenhangs der Praktikumsvariablen mit der Hochschule bzw. dem angestrebten Lehramtszugang zeigen, dass sowohl bei den Tätigkeiten im Praktikum $\left(M_{I C C}=0,15, S D_{I C C}=0,10\right)$, als auch bei der Betreuung $\left(M_{I C C}=0,34, S D_{I C C}=0,17\right)$ Zusammenhänge mit der Hochschule bestehen, nicht jedoch mit dem Lehramtszugang (Tätigkeiten: $M_{I C C}=0,01$, $S D_{I C C}=0,01$; Betreuung: $\left.M_{I C C}=0,03, S D_{I C C}=0,02\right)$.

Vergleichsgruppe Die Vergleichsstichprobe umfasst 527 Lehramtsstudierende (65\% weiblich; Alter: $M=21,3$ Jahre, $S D=3,9$ ), die ihr Studium ebenfalls zum Wintersemester 2009/10 bzw. 2010/11 an denselben Universitäten aufnahmen, jedoch in diesem Zeitraum kein Praktikum absolvierten. Von diesen Studierenden streben $79 \%(n=415)$ das Gymnasium und 9\% $(n=46)$ die Grundschule als Lehramtszugang an.

Dropout-Analysen Zum zweiten Messzeitpunkt nahmen in der Praktikumsgruppe 296 Studierende teil und zum dritten Messzeitpunkt noch 205, das entspricht einem Rücklauf von 69,5\%. Zwischen dem ersten und zweiten Messzeitpunkt kann kein Dropout berichtet werden, da nur Studierende mit Angaben zu einem absolvierten Praktikum in die untersuchte Stichprobe aufgenommen wurden. In der Vergleichsstichprobe liegt der Rücklauf bei 99,4\% zum zweiten bzw. 69,6\% über drei Messzeitpunkte $\left(n_{T 1}=527 ; n_{T 2}=524 ; n_{T 3}=360\right)$. Diese Rücklaufquoten liegen deutlich über denen vergleichbarer Studien (55\%, Schüle et al. 2017; $63 \%$, Malmberg 2008). Anschließende $t$-Tests für unabhängige Stichproben zwischen den Studierenden, deren Angaben nicht bzw. auch zum dritten Messzeitpunkt vorlagen, hinsichtlich der abhängigen Variablen zeigen, dass es weder in Praktikumsgruppe (Selbstkonzept Fach: $M_{\text {prakt }}=3,04$ vs. $M_{\text {Drop }}=2,92, t(293)=1,93$, n. s., $d=0,24$; Selbstkonzept Erziehung: $M_{\text {prakt }}=3,29$ vs. $M_{\text {Drop }}=3,34, t(293)=-0,66$, n. s., $d=0,08$; Berufswahlsicherheit: $M_{\text {prakt }}=3,31$ vs. $M_{\text {Drop }}=3,21, t(294)=1,45$, n. s., $\left.d=0,18\right)$ noch in der Vergleichsgruppe (Selbstkonzept Fach: $M_{\text {prakt }}=3,13$ vs. $M_{\text {Drop }}=3,06, t(515)=1,38$, n. s., $d=0,13$; Selbstkonzept Erziehung: $M_{\text {prakt }}=3,24$ vs. $M_{\text {Drop }}=3,28, t(515)=-0,94$, n. s., $d=0,09$; Berufswahlsicherheit: $M_{\text {prakt }}=3,31$ vs. $M_{\text {Drop }}=3,18, t(525)=2,89, p<0,05$, $d=0,27$ ) bedeutsame systematische Unterschiede gibt.

\subsection{Instrumente}

Berufsbezogenes Selbstkonzept Das Selbstkonzept wurden mit den zwei Subskalen „Fach“ und „Erziehung“ des Instruments „Zur Erfassung berufsbezogener Selbstkonzepte von Lehramtsstudierenden (ERBSE-L)“ erfasst (Retelsdorf et al. 2014). Dieses Instrument erfasst multidimensional spezifische Selbstkonzepte für das Lehramt. Alle Skalen wurden zu zwei Messzeitpunkten erhoben, zu T1 zu Beginn des Studiums und zu T3 ca. drei Monate nach dem Praktikum. Die Items 
Tab. 1 Deskriptive Analysen und Itembeispiele der verwendeten Instrumente

\begin{tabular}{|c|c|c|c|c|c|}
\hline & Items & $M$ & $S D$ & Cronb. $\alpha$ & Itembeispiel \\
\hline \multicolumn{6}{|c|}{ Vergleichsgruppe ohne Praktikum } \\
\hline Fach zu T1 & \multirow[t]{2}{*}{3} & 3,11 & 0,49 & 0,75 & \multirow{2}{*}{$\begin{array}{l}\text { „Ich denke, meine Studienfächer } \\
\text { liegen mir besonders“" }\end{array}$} \\
\hline Fach zu T3 & & 2,95 & 0,53 & 0,84 & \\
\hline Erziehung zu T1 & \multirow[t]{2}{*}{4} & 3,25 & 0,47 & 0,77 & \multirow{2}{*}{$\begin{array}{l}\text { „Mir liegt es, Kinder zu } \\
\text { motivieren“ }\end{array}$} \\
\hline Erziehung zu T3 & & 3,31 & 0,50 & 0,81 & \\
\hline Berufswahlsicherheit T1 & \multirow{3}{*}{4} & 3,27 & 0,47 & 0,64 & \multirow{3}{*}{$\begin{array}{l}\text { „Ich bin mir sicher, dass ich das } \\
\text { Lehramtsstudium erfolgreich } \\
\text { beenden werde“ }\end{array}$} \\
\hline Berufswahlsicherheit T2 & & 3,17 & 0,54 & 0,73 & \\
\hline Praktikumsgruppe & & & & & \\
\hline Fach zu T1 & \multirow[t]{2}{*}{3} & 3,00 & 0,49 & 0,77 & \\
\hline Fach zu T3 & & 2,86 & 0,51 & 0,84 & \\
\hline Erziehung zu T1 & \multirow[t]{2}{*}{4} & 3,31 & 0,45 & 0,72 & \\
\hline Erziehung zu T3 & & 3,34 & 0,49 & 0,81 & \\
\hline Berufswahlsicherheit T1 & \multirow{2}{*}{4} & 3,28 & 0,48 & 0,66 & \\
\hline Berufswahlsicherheit T2 & & 3,30 & 0,48 & 0,64 & \\
\hline \multicolumn{6}{|l|}{ Praktikum } \\
\hline Schulerfahrung & 4 & 3,10 & 0,50 & 0,62 & $\begin{array}{l}\text { „... habe ich viel über das Unter- } \\
\text { richten gelernt““ }\end{array}$ \\
\hline Berufswahlreflexion & 3 & 3,52 & 0,47 & 0,63 & $\begin{array}{l}\text { „... wusste ich danach, dass der } \\
\text { Lehrerberuf zu mir passt“ }\end{array}$ \\
\hline Theorie-Praxis-Transfer & 2 & 1,97 & 0,72 & 0,77 & $\begin{array}{l}\text { „... konnte ich das im Studium } \\
\text { Gelernte umsetzen“ }\end{array}$ \\
\hline Betreuungsqualität & $4^{\mathrm{a}}$ & 2,91 & 0,72 & & $\begin{array}{l}\text { „An der Uni: individuelle Be- } \\
\text { treuung, z. B. durch Dozenten, } \\
\text { Tutoren etc.“ }\end{array}$ \\
\hline \multicolumn{6}{|l|}{ Persönlichkeit } \\
\hline Gewissenhaftigkeit & 4 & 3,34 & 0,52 & 0,78 & „Offen für neue Erfahrungen“ \\
\hline Extraversion & 3 & 2,86 & 0,67 & 0,80 & ,Zurückhaltend“ (-) \\
\hline Neurotizismus & 3 & 1,96 & 0,48 & 0,63 & $\begin{array}{l}\text { „Leicht aus der Fassung zu brin- } \\
\text { gen“ }\end{array}$ \\
\hline
\end{tabular}

${ }^{a}$ Mittlere Zusammenfassung verschiedener Facetten der wahrgenommenen Betreuungsqualität

wurden auf einem vierstufigen Antwortformat $(1=$,trifft überhaupt nicht zu“ bis $4=$,,trifft völlig zu“") erhoben (Kennwerte und Itembeispiele s. Tab. 1).

Sicherheit der Berufswahl Die „Sicherheit der Berufswahl“ wurde mit fünf Items und einem vierstufigen Antwortformat ( $1=$,trifft überhaupt nicht $\mathrm{zu}$ “ bis $4=$,trifft völlig zu“) erfasst. Die Skala wurde zu zwei Messzeitpunkten erhoben, einmal zu Beginn des Studiums (T1) und zu Beginn des zweiten Semesters (T2) (s. Tab. 1). Somit wurde die Berufswahlsicherheit zum gleichen Zeitpunkt erhoben, wie die Einschätzungen zum Praktikum. Während sich die Fragen zum Praktikum retrospektiv auf das bereits absolvierte Praktikum richteten, wurde die Berufswahlsicherheit aktuell zum Befragungszeitpunkt erfasst.

Hospitationspraktikum Die Lerngelegenheit Praktikum wurde über die selbsteingeschätzte Erreichung der Aufgaben des Praktikums operationalisiert. Es handelt 
sich hierbei um Aufgaben, die das Praktikum als Lerngelegenheit erfüllen soll, nicht um (Arbeits-)Aufgaben, die an die Studierenden gerichtet sind. Es wurde auf die in der Literatur und den universitären Curricula beschriebenen Ziele „Berufswahlreflexion“, „Theorie-Praxis-Transfer“ und den „Erfahrungen im schulischen Kontext“ (vgl. Terhart 2002) zurückgegriffen und in Anlehnung an Flagmeyer und HoppeGraff (2006) 10 Items konstruiert. Erfasst wurden diese ebenfalls mittels eines vierstufigen Antwortformats ( $1=$,trifft überhaupt nicht zu“ bis $4=$,trifft völlig zu“). Intendiert war die Konstruktion von drei Skalen, die Faktorenstruktur wurde mittels Exploratorischer Strukturgleichungsmodelle (ESEM) geprüft. Bei diesem Verfahren wird eine exploratorische Faktoranalyse (EFA) um konfirmatorische (KFA) Elemente erweitert. Dieses Verfahren wurde gewählt, da es sich nicht um etablierte Skalen, sondern um Eigenkonstruktionen handelt. Es konnten die postulierten drei Faktoren identifiziert werden, ein Item $(,, \ldots$ wusste ich danach, dass meine Fächer zu mir passen“) wurde aufgrund von Mehrfachladungen aus weiteren Analysen ausgeschlossen. Die Kennwerte des Messmodells sind als gut zu bewerten $\left(\chi^{2}(8)=36,31\right.$, $p<0,01, \mathrm{CFI}=0,963$, RMSEA =0,060, SRMR =0,027).

Die wahrgenommene Betreuungsqualität im Praktikum wurde mittels vierstufigen

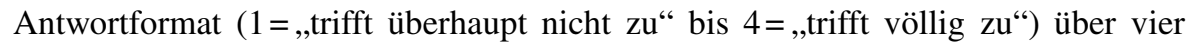
Items erfasst, bei denen die Studierenden einschätzen sollten, wie hilfreich sie die Betreuung an Hochschule und Schule empfanden. Diese vier Items wurden zu einem Item zusammengefasst, in welchem die erlebte Qualität aller Betreuungsvarianten gemittelt ist.

Alle Merkmale des Hospitationspraktikums wurden zu T2 erhoben. Die Kennwerte sowie Itembeispiele aller verwendeten Konstrukte sind in Tab. 1 dargestellt.

Persönlichkeit Es wurden die für praktische Lerngelegenheiten prominent beschriebenen Facetten der Big Five der Persönlichkeit „Gewissenhaftigkeit“, „Neurotizismus (Emotionale Instabilität)“ und „Extraversion“ als individuelle Determinanten sowohl der Wahrnehmung der Betreuungsqualität, als auch der Bewertung der Lerngelegenheit Praktikum kontrolliert. Erfasst wurden sie zu T1 mittels einer Kurzversion von drei bis vier Items, die auf einer Skala von 1 (,stimmt gar nicht“) bis 4 (,stimmt genau“) zu beantworten waren (Herzberg und Brähler 2006).

Das Messmodell zeigt eine gute Anpassung an die Daten $\left(\chi^{2}(32)=61,136\right.$, $p<0,01, \mathrm{CFI}=0,952, \mathrm{RMSEA}=0,058, \mathrm{SRMR}=0,054)$. Ein Item der Skala ,Neurotizismus“ musste jedoch aufgrund schlechter Passung aus den Analysen ausgeschlossen werden (Item ,ängstlich“).

\subsection{Auswertung}

Die Überprüfung der Fragestellungen erfolgte zweischrittig. Zunächst wurden zur Beantwortung der ersten Forschungsfrage multiple Gruppenvergleiche zur Absicherung der Praktikumseffekte zwischen der Praktikumsgruppe und der Vergleichsgruppe ohne Praktikum durchgeführt. Es wurde geprüft, ob es Unterschiede im Selbstkonzept und der Berufswahlsicherheit in Abhängigkeit der Gruppenzugehörigkeit gibt. Im zweiten Schritt wurden anhand der Praktikumsgruppe Strukturgleichungsmodelle zur Beantwortung der Forschungsfrage 2 berechnet. 
Die deskriptiven Angaben und Cronbachs $\alpha$ der Tab. 1 wurden mit SPSS 23 berechnet. Alle Berechnungen der Struktur- und Messmodelle wurden mit der Software Mplus 7.11 (Muthén und Muthén 2013) durchgeführt. Dabei gingen alle verfügbaren Informationen, auch solche von Personen mit fehlenden Werten, in die Modellschätzung ein. Dazu wurde der Full Information Maximum Likelihood (FIML) Ansatz zur Schätzung von Modellen mit unvollständigen Daten genutzt. Dieses Vorgehen verhindert auch bei größeren Ausfallraten Verzerrungen, wie sie durch einen fallweisen Ausschluss von Personen mit fehlenden Werten entstehen würden (Schafer und Graham 2002).

Der hierarchischen Datenstruktur (Studierende in Universitäten) wurde durch die Anwendung des entsprechenden Schätzverfahrens Rechnung getragen. Die Modellparameter wurden dazu über den MLR-Algorithmus unter der Analyseoption type $=$ complex geschätzt. Bei diesem Schätzverfahren werden die Standardfehler für die Verletzung der Unabhängigkeitsannahme korrigiert. Ohne diese Korrektur kann es zu einer Überschätzung der Signifikanzen kommen (Hox 2002). Die Intraklassenkorrelation (ICC) fällt in der vorliegenden Studie unterschiedlich aus. Bei den Variablen des Gruppenvergleichs liegen sie bei $M_{I C C}=0,02\left(S D_{I C C}=0,01\right)$, im Strukturmodell der Praktikumsgruppe bei $M_{I C C}=0,08\left(S D_{I C C}=0,16\right)$. Hohe ICCs liegen bei den Variablen „Dauer des Praktikums“ $r_{I C C}=0,65$, der ,wahrgenommenen Betreuungsqualität" $r_{I C C}=0,11$ und im Selbstkonzept Fach vor (T1: $r_{I C C}=0,062$; T3: $\left.r_{I C C}=0,078\right)$, bei allen weiteren Variablen liegt sie unter $r_{I C C}<0,06$.

Als zentrale Voraussetzung für multiple Gruppenvergleiche wurde die skalare Messinvarianz zwischen den Gruppen und longitudinal geprüft. Als Voraussetzung der Strukturmodelle innerhalb der Praktikumsgruppe wurde das Vorliegen metrischer longitudinaler Messinvarianz geprüft (Kleinke et al. 2017). Zur Einschätzung der Modelle wurden die FIT-Statistiken hinzugezogen, dabei sprechen Änderungen im $\mathrm{CFI}<0,01$ und des RMSEA $<0,015$ gegen einen Unterschied in den Modellen (Chen 2007). Die Analysen zeigen, dass die jeweiligen Messinvarianzbedingungen und somit die Voraussetzungen zur Prüfung von Gruppenunterschieden bzw. der Berechnung des Strukturmodells hinreichend erfüllt sind (s. Onlinematerial 1).

Im Strukturmodell wurden Korrelationen (s. Tab. 2) zwischen den Konstrukten eines Messzeitpunkts ebenso zugelassen wie die Fehlerautokorrelationen der Selbstkonzeptindikatoren im Messmodell.

\section{Ergebnisse}

\subsection{Deskriptive Befunde}

In Tab. 1 sind die Mittelwerte und Standardabweichungen dargestellt. Insgesamt zeigt sich, dass die Mittelwerte der erhobenen Selbstkonzepte zu Fach und Erziehung sowie der Sicherheit der Berufswahl bei beiden Teilstichproben im deutlich zustimmenden Bereich der Ratingskala liegen.

Ebenso zeigen die Aufgaben „Erfahrungen im schulischen Kontext“ und „Berufswahlreflexion“ einen hohen Mittelwert. Die Studierenden der Praktikumsgruppe geben demnach an, schulische Erfahrungen gesammelt und mit dem Ergebnis ei- 


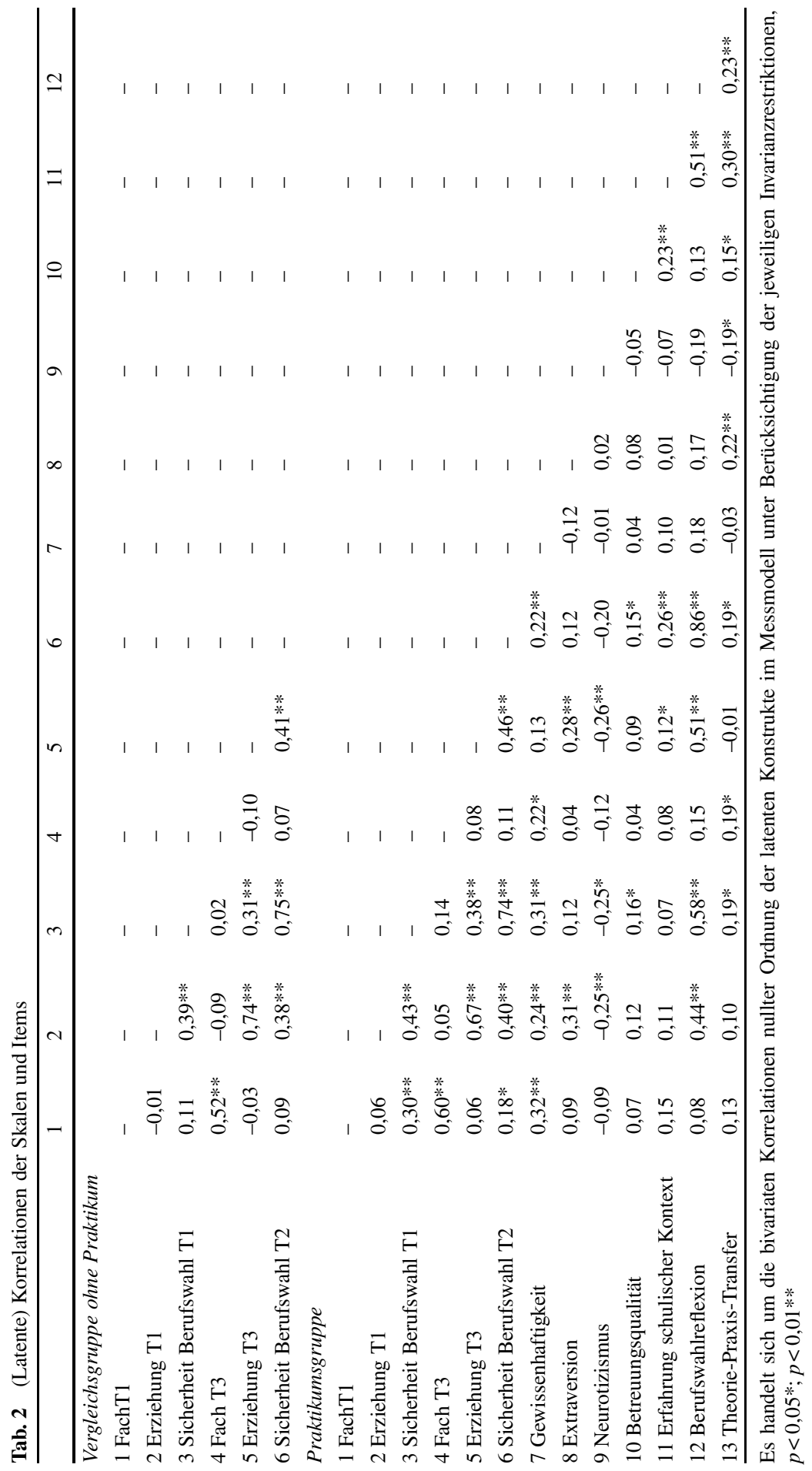


ner Bestätigung über ihre Berufswahl reflektiert zu haben. Der Bereich „TheoriePraxis-Transfer“" wird im Gegensatz dazu im ablehnenden Teil der Skala beantwortet. Die Betreuungsqualität wird bei einem Mittelwert von 2,91 $(S D=0,72)$ eher positiv wahrgenommen. Anhand der Intraklassenkorrelation $\left(r_{I C C}=0,11\right)$ ist von einer Abhängigkeit der Hochschule, nicht jedoch vom Lehramtszugang $\left(r_{I C C}=0,008\right)$ auszugehen.

\subsection{Gruppenvergleich zwischen Praktikums- und Vergleichsgruppe}

Zur Beantwortung der ersten Forschungsfrage wurden die Mittelwertsunterschiede zwischen den Gruppen in einem Mehrgruppenmodell auf Signifikanz getestet. Die Mittelwerte der Vergleichsgruppe wurden dabei auf null fixiert (Referenzgruppe) und die Differenzen zur Praktikumsgruppe geschätzt. Die Werte der Praktikumsgruppe können entsprechend als latente Mittelwertsdifferenzen interpretiert werden (Kleinke et al. 2017).

Das Mehrgruppenmodell zeigt bei Berücksichtigung aller Restriktionen der Messinvarianzprüfungen einen akzeptablen Fit $(\chi 2(512)=918,41, p<0,01, \mathrm{CFI}=0,927$, RMSEA = 0,044, SRMR = 0,09).

Die Betrachtung der geschätzten Mittelwertsdifferenzen verdeutlicht, dass es vor dem Praktikum keine Unterschiede gab $\left(\Delta M_{\text {Fach }}=-0,08, p=0,31 ; \Delta M_{\text {Erziehung }}=0,12\right.$, $\left.p=0,12 ; \Delta M_{\text {Berufswahlsicherheit }}=-0,01, p=0,93\right)$, jedoch lassen sich nach dem Praktikum bedeutsame Gruppenunterschiede in allen abhängigen Variablen beobachten $\left(\Delta M_{\text {fach }}=-0,44^{* *}, p<0,01 ; \Delta M_{\text {Erziehung }}=0,19 *, p<0,05 ; \Delta M_{\text {Berufswahlsicherheit }}=0,25^{* *}\right.$, $p<0,01)$. Das Selbstkonzept zum Fach ist zum dritten Messzeitpunkt in der Praktikumsgruppe somit signifikant niedriger, die Sicherheit der Berufswahl und das Selbstkonzept zur Erziehung signifikant höher, als in der Vergleichsgruppe.

\subsection{Entwicklung der Kompetenzfacetten in der Praktikumsgruppe}

Die erwarteten Beziehungen zwischen den Modellvariablen wurden mit Hilfe von Strukturgleichungsmodellen überprüft. Das geprüfte Modell ist in Abb. 1 dargestellt, die Pfadkoeffizienten lassen sich der Tab. 3 entnehmen. Aus Vereinfachungsgründen wird nur das Struktur- und nicht das Messmodell dargeboten, sowie auf Angabe der Kovarianzen verzichtet.

Das den theoretischen Annahmen folgende Modell weist eine akzeptable Anpassungsgüte auf: $\chi 2(852)=1154,55, p<0,01, \mathrm{CFI}=0,911, \mathrm{RMSEA}=0,035$, $\mathrm{SRMR}=0,075$.

Die Vorhersage der Selbstkonzepte zu T3 bzw. der Berufswahlsicherheit zu T2 durch die Messung zum ersten Messzeitpunkt dient der Kontrolle der Ausgangswerte. Die Höhe der Vorhersage $(\beta=0,52 * * / 0,52 * *$, s. Tab. 3) lässt auf eine grundsätzliche Stabilität der Selbstkonzeptfacetten über das erste Studienjahr schließen. Die Sicherheit der Berufswahl zeigt sich - bei Berücksichtigung aller weiteren Faktoren im Strukturmodell - mit $\beta=0,31 * *$ deutlich weniger stabil. Hier deutet sich bereits ein starker Effekt der Lerngelegenheit Praktikum an, da die Korrelation Nullter Ordnung bei $r=0,74$ liegt. 
Tab. 3 Ergebnisse des Strukturgleichungsmodells zur Vorhersage der Kompetenzentwicklung durch das Hospitationspraktikum

\begin{tabular}{|c|c|c|c|c|c|c|c|}
\hline \multirow[b]{2}{*}{ Prädiktoren } & \multicolumn{4}{|l|}{$\mathrm{T} 2$} & \multicolumn{3}{|c|}{$\mathrm{T} 3$} \\
\hline & $\begin{array}{l}\text { Wahrg. } \\
\text { Betreu- } \\
\text { ung }\end{array}$ & $\begin{array}{l}\text { Erfahrung } \\
\text { in der } \\
\text { Schule }\end{array}$ & $\begin{array}{l}\text { Berufswahl- } \\
\text { reflexion }\end{array}$ & $\begin{array}{l}\text { Theorie- } \\
\text { Praxis- } \\
\text { Transfer }\end{array}$ & $\begin{array}{l}\text { Sicherheit } \\
\text { Berufs- } \\
\text { wahl }\end{array}$ & $\begin{array}{l}\text { SK } \\
\text { Fach }\end{array}$ & $\begin{array}{l}\text { SK } \\
\text { Erz }\end{array}$ \\
\hline \multicolumn{8}{|c|}{ Kontrollvariablen T1: } \\
\hline Extraversion & $-0,07$ & 0,05 & 0,17 & $0,21 *$ & - & - & - \\
\hline Gewissenhaftigkeit & $-0,02$ & 0,09 & $-0,02$ & $-0,06$ & - & - & - \\
\hline Neurotizismus & $-0,19^{*}$ & $-0,05$ & $-0,65 * *$ & $-0,16$ & - & - & - \\
\hline $\begin{array}{l}\text { Sicherheit Berufs- } \\
\text { wahl }\end{array}$ & - & - & - & - & $0,31 * *$ & - & - \\
\hline SK Fach & - & - & - & - & - & $0,52 * *$ & - \\
\hline SK Erziehung & - & - & - & - & - & - & $0,52 * *$ \\
\hline \multicolumn{8}{|l|}{$T 2:$} \\
\hline Wahrg. Betreuung & - & $0,22 * *$ & 0,01 & $0,14 *$ & - & - & - \\
\hline $\begin{array}{l}\text { Erfahrung in } \\
\text { Schule }\end{array}$ & - & - & - & - & $-0,08$ & $-0,07$ & 0,08 \\
\hline $\begin{array}{l}\text { Berufswahl- } \\
\text { reflexion }\end{array}$ & - & - & - & - & $0,54 * *$ & 0,08 & $0,22 *$ \\
\hline $\begin{array}{l}\text { Theorie-Praxis- } \\
\text { Transfer }\end{array}$ & - & - & - & - & $-0,02$ & 0,10 & $-0,13 *$ \\
\hline$R^{2}$ & 0,04 & 0,07 & 0,44 & 0,09 & 0,54 & 0,29 & 0,41 \\
\hline
\end{tabular}

Alle Parameterschätzungen in standardisierter Form. Aus Darstellungsgründen wird auf die Angabe der Kovarianzen verzichtet

T1 Messzeitpunkt 1, T2 Messzeitpunkt 2, T3 Messzeitpunkt 3, SK Fach Selbstkonzept Fach, SK Erz Selbstkonzept Erziehung

$* p<0,05 ; * * p<0,01$

Zur Beantwortung der zweiten Forschungsfrage wird der Fokus auf die Prädiktion der Selbstkonzepte zu Fach und Erziehung sowie der Berufswahlsicherheit durch das Praktikum gelegt. Es zeigt sich, dass die „Berufswahlreflexion“ einen positiven Beitrag zum Selbstkonzept Erziehung $\left(\beta=0,22^{*}\right)$ und einen bedeutenden Beitrag zur Berufswahlsicherheit $\left(\beta=0,54^{* *}\right)$ leistet. Wenn das bisherige Wissen aus dem Studium auf die Praxis übertragen werden kann (,Theorie-Praxis-Transfer“), so nimmt dies wiederum einen negativen Einfluss auf das Selbstkonzept Erziehung $\left(\beta=-0,13^{*}\right)$.

Die wahrgenommene Betreuungsqualität nimmt Einfluss auf die Einschätzung der Praktikumsaufgaben „Erfahrungen in der Schule“ $(\beta=0,22 * *)$ und „TheoriePraxis-Transfer“ $\left(\beta=0,14^{*}\right)$, nicht jedoch auf die „Berufswahlreflexion“ $(\beta=0,01$, $p=0,94)$.

Weiterhin wurde der Einfluss von Persönlichkeitsmerkmalen auf die Einschätzung der Praktikumsaufgaben und der Wahrnehmung der Betreuungsqualität kontrolliert. Das Persönlichkeitsmerkmals „Neurotizismus“ prädiziert sowohl einen hohen negativen Effekt auf die ,Berufswahlreflexion“ $\left(\beta=-0,65^{* *}\right)$, als auch einen negativen Effekt auf die wahrgenommene Qualität der Betreuung $\left(\beta=-0,19^{*}\right)$. Ansonsten leistet nur Extraversion eine positive Vorhersage für den Theorie-Praxis-Transfer $\left(\beta=0,21^{*}\right)$. 


\section{Diskussion}

Zusammengefasst zeigen die Ergebnisse der vorliegenden Studie, dass das Praktikum (s)einen Beitrag zur Berufsorientierung leistet: der Gruppenvergleich deckt Unterschiede nach dem Praktikum auf, Analysen zur Lerngelegenheit zeigen, dass die curricular intendierten Aufgaben des Praktikums Einfluss auf das Selbstkonzept und die Berufswahlsicherheit nehmen.

Der Gruppenvergleich zwischen Studierenden mit und ohne absolviertem Hospitationspraktikum deckt Unterschiede in den abhängigen Variablen nach dem Praktikum auf, die vor dem Praktikum nicht beobachtet werden konnten. Schon kurzzeitige Hospitationspraktika nehmen somit Einfluss auf die Veränderung des Selbstkonzeptes und der Berufswahlsicherheit. Die Effekte sind insgesamt gering, was bei der Höhe der Selbsteinschätzungen im Ausgangswert kaum überrascht (vgl. Rothland und Straub 2018). Vergleichbare Studien zu den berufsbezogenen Selbstkonzepten oder zum fachlichen Selbstkonzept berichten ähnlich hoch ausgeprägte Selbsteinschätzungen (Klostermann et al. 2014; Retelsdorf et al. 2014; Rothland und Straub 2018). Erste längsschnittliche Ergebnisse zur Veränderung berufsbezogener Selbstkonzepte durch Praxissemester berichten, ähnlich zu den Befunden der vorliegenden Studie, signifikante aber geringe Effekte (Rothland und Straub 2018).

In der Praktikumsgruppe ist das fachliche Selbstkonzept nach dem Praktikum niedriger, das erzieherische Selbstkonzept und die Berufswahlsicherheit jedoch höher, als in der Vergleichsgruppe. Das mittlere fachliche Selbstkonzept sinkt zwar in beiden Gruppen, die Mittelwertsdifferenz zeigt jedoch ein vergleichsweise niedrigeres Selbstkonzept in der Praktikumsgruppe. Dies legt die Interpretation nahe, dass durch das Praktikum eine kritische Betrachtung der eigenen fachlichen Fähigkeiten erreicht werden kann. Das ist angesichts der hohen Eingangswerte wünschenswert. Empirische Forschung zum Selbstkonzept legt nahe, dass ein realistisches Selbstkonzept einem (über)hohen vorzuziehen sei, um das Risiko einer Selbstüberschätzung zu vermeiden. In Anlehnung an Befunde im schulischen Bereich tendieren Personen mit einem hohen fachbezogenen Selbstkonzept dazu, nicht mehr viel Anstrengung in das Fach zu investieren (Blanton et al. 1999). Die mögliche Folge für das weitere Lehramtsstudium wäre ein vermindertes Interesse an den theoretischen Ausbildungsteilen und damit ein Auseinanderdriften von Theorie und Praxis.

Im Gegensatz zum fachlichen bleibt das erzieherische Selbstkonzept auf hohem Niveau stabil und zeigt nur einen geringen Unterschied zwischen den Gruppen. Ein "Gesundschrumpfen“ dieser hohen Selbsteinschätzung scheint im Hospitationspraktikum nicht erreicht werden zu können. Gröschner et al. (2013) beobachten bei ihren Analysen zur Kompetenzentwicklung im Praxissemester ebenfalls, dass die geringste Veränderung im Bereich „Erziehen“ berichtet wird. Das Selbstkonzept Erziehung ist zudem sehr allgemein auf einen positiven Umgang mit Kindern und Jugendlich formuliert und richtet sich nicht auf konkrete Tätigkeiten oder Situationen. Ein kurzfristiges Hospitationspraktikum scheint dementsprechend keine Unterschiede stiften zu können.

Die Berufswahlsicherheit bleibt in der Praktikumsgruppe ebenfalls eher stabil und sinkt in der Vergleichsgruppe leicht ab. Die hohe Stabilität der Berufswahlsicherheit entspricht ebenfalls bisher bekannten Befunden (Bauer et al. 2011, Seifert und 
Schaper 2018). Die Ergebnisse der vorliegenden Studie zeigen jedoch auch, dass ein frühes Praktikum einem Absinken der Berufswahlsicherheit entgegenwirken kann. Vergleichbare Ergebnisse zum Praxissemester zeigen, dass zwar ein Absinken der Berufswahlmotivation über die ersten Semester beobachtet wird, jedoch ein Anstieg und die Stabilisierung durch das Praxissemester erreicht werden kann (Holtz 2014). Zu vermuten wäre, dass durch den, bei den Studierenden beliebten, Praxisund Berufsfeldkontakt eine Stabilisierung und die Bestätigung, das richtige Fach zu studieren, auch zu früheren Zeitpunkten im Studium erreicht werden kann. Diese Bestätigung bzw. die Sicherheit der (richtigen) Berufswahl führt im weiteren Verlauf des Studiums nach bisheriger Befundlage zur Aufrechterhaltung der Motivation und des Studieninteresses (Rösler et al. 2013; van der Veen et al. 2005).

Es muss an dieser Stelle jedoch kritisch angemerkt werden, dass es sich bei der Vergleichsgruppe nicht um eine Kontrollgruppe im strengen Sinne handelt. Es kann dementsprechend nicht abschließend geklärt werden, ob der Nicht-Absolvierung des Praktikums zu diesem Zeitpunkt Gründe vorausgehen, die auch Einfluss auf die Entwicklung des Selbstkonzepts und der Berufswahlsicherheit nehmen. Weiterhin handelt es sich bei den Einschätzungen der Kompetenzfacetten um Selbsteinschätzungen. Dies bedeutet einerseits, dass anhand der zugeschriebenen Kompetenzeinschätzung und -entwicklung von Selbstüberschätzungen ausgegangen werden kann (zsf. Hascher 2012), die hier untersuchten Selbstkonzepte sind jedoch per Definition selbstbezogene Kognitionen (z.B. Möller und Trautwein 2009; Retelsdorf et al. 2014) und können anders als wissensbezogene Kompetenzen nicht via Test als Fremdeinschätzung erfasst werden.

Die Ergebnisse zur Entwicklung der Kompetenzfacetten innerhalb der Praktikumsgruppe fallen je nach abhängiger Variable unterschiedlich aus. Die größte Aufklärung kann durch die Berufswahlreflexion erreicht werden. Wenn mit dem Ergebnis der Bestätigung der Berufswahl reflektiert wird, beeinflusst dies die eigene Berufswahlsicherheit und das Selbstkonzept zur Erziehung. Der Effekt auf die Berufswahlsicherheit ist sogar höher, als die longitudinale Stabilität der Variable. Dies bedeutet aber auch, dass Studierende ihre Berufswahl eher bestätigen, statt kritisch die persönliche Eignung zu prüfen. Durch die Stärkung der persönlichen Sicherheit der (richtigen) Berufswahl könnte ein Praktikum frühen Studienabbruchintentionen entgegenwirken, insgesamt ist jedoch nur wenig über den Zusammenhang von Studienabbruch und Praktikumserfahrungen bekannt (vgl. Rothland und Boecker 2015). Die Wirkung von Praktikumserfahrungen auf den weiteren Studienverlauf sollte in weiteren Studien geprüft werden. Grundsätzlich legen vergleichbare Studien jedoch nahe (bspw. Schubarth et al. 2014), dass Praxisphasen für die Berufsorientierung der Studierenden durch die Reflexion der erlebten Praxis förderlich sind.

Eine Zustimmung zum Theorie-Praxis-Transfer im Sinne der Anwendung von bisher Gelerntem wirkt sich interessanterweise negativ, die Bestätigung des Berufswunsches hingegen positiv auf das Selbstkonzept zur Erziehung aus. Auf der einen Seite scheint demnach ein Transfer in der gewünschten Form zu passieren: angewandtes Wissen führt zur kritischeren Wahrnehmung selbstzugeschriebener Kompetenzen. Auf der anderen Seite führt die positive Berufswahlreflexion zur Erhöhung selbsteingeschätzter erzieherischer Kompetenzen. Es ist zu vermuten, dass allein die Bestätigung des Berufswunsches schon zur Selbstzuschreibung berufsbezogener 
Kompetenzen führt. Bisherige empirische Befunde zeigen diesen Effekt für unterrichtliches Handeln im Sinne von ,ich mache, also kann ich“ (Arnold et al. 2011; Hascher 2011). Ähnlich kritische Befunde zum Theorie-Praxis-Transfer berichten auch Schubarth et al. (2014) zum Praxissemester in Potsdam, bei dem ein Praxistransfer wohl grundsätzlich gelingt, die Reflexion der Erfahrungen jedoch von den Studierenden als mangelhaft eingeschätzt wird.

Während die Veränderung der Berufswahlsicherheit und des Selbstkonzepts Erziehung durch die Aufgaben des Praktikums vorhergesagt werden können, gelingt dies beim fachlichen Selbstkonzept nicht. Aufgrund der beobachteten Gruppenunterschiede kann vermutet werden, dass weitere nicht erfasste Praktikumsvariablen Einfluss nehmen.

Je hilfreicher die Betreuung wahrgenommen wird, desto eher werden Erfahrungen in der Schule gesammelt und der Theorie-Praxis-Transfer gelingt, wobei die Betreuung insgesamt in hohem Maß von der Hochschule abhängig ist. Auf die Berufswahlreflexion nimmt die Betreuung jedoch keinen Einfluss. Das ist angesichts des großen Einflusses des Persönlichkeitsmerkmals Neurotizismus problematisch. Einerseits wirkt sich Neurotizismus negativ auf die Reflexion aus, d.h. Personen mit einer hohen Ausprägung dieses Merkmals reflektieren dementsprechend nicht positiv über ihre Berufswahl und können daher die positive Folge der Berufswahlreflexion, die höhere Berufswahlsicherheit, nicht nutzen. Auf der anderen Seite führt Neurotizismus auch zu einer negativen Betreuungswahrnehmung. Dieses Ergebnis zeigt die Notwendigkeit der Berücksichtigung individueller Merkmale, um die Lerngelegenheit Praktikum so zu gestalten, dass sie lernwirksam wird. Dieser Aspekt stellt insbesondere die Bedeutung der Schulung betreuender Dozenten/innen und Mentoren/innen heraus (Hascher und Moser 2001; Lazarides und Mohr 2015).

Durch das Prä-Post-Design und den Einbezug einer Vergleichsgruppe ohne Praktikum erweitert die vorliegende Studie bisherige Erkenntnisse zu Praxisphasen. Es kann gezeigt werden, dass dem Hospitationspraktikum insbesondere für die Aufrechterhaltung und Entwicklung der Berufswahlsicherheit eine wichtige Rolle zukommt. Die gefundenen längsschnittlichen Effekte reihen sich in bisher bekannte Ergebnisse zum Praxissemester ein, trotz einer zeitlich deutlich geringeren Dauer des hier untersuchten Hospitationspraktikums. Diese Befunde stützen die Diskussion um die Ausgestaltung von Praxisphasen, da die Dauer der Praxisphase keinen bedeutenden Einfluss zu haben scheint (Gröschner und Müller 2014). Weiterhin stärkt die vorliegende Studie die Ergebnisse zur Bedeutung der Betreuung. Daneben zeigen sich individuelle Determinanten als wichtige Faktoren zur Nutzung der Lerngelegenheit Hospitationspraktikum und sollten bei der Ausgestaltung Berücksichtigung finden.

Förderung Die Daten für die hier präsentierten Analysen stammen aus dem Projekt „PaLea - Panel zum Lehramtsstudium“, gefördert vom Bundesministerium für Bildung und Forschung (FKZ: 01PH08004/01PH13001/01PH16001).

Open Access Dieser Artikel wird unter der Creative Commons Namensnennung 4.0 International Lizenz (http://creativecommons.org/licenses/by/4.0/deed.de) veröffentlicht, welche die Nutzung, Vervielfältigung, Bearbeitung, Verbreitung und Wiedergabe in jeglichem Medium und Format erlaubt, sofern Sie den/die ursprünglichen Autor(en) und die Quelle ordnungsgemäß nennen, einen Link zur Creative Commons Lizenz beifügen und angeben, ob Änderungen vorgenommen wurden. 


\section{Literatur}

Arnold, K.-H., Hascher, T., Messner, R., Niggli, A., Patry, J.-L., \& Rahm, S. (2011). Empowerment durch Schulpraktika. Perspektiven wechseln in der Lehrerbildung. Bad Heilbrunn: Julius Klinkhardt.

Bach, A., Besa, K.-S., \& Arnold, E. (2014). Bedingungen von Lernprozessen in Schulpraktika: Ergebnisse aus dem Projekt ESIS (Entwicklung Studierender in Schulpraktika). In K.-H. Arnold, A. Gröschner \& T. Hascher (Hrsg.), Schulpraktika in der Lehrerbildung. Theoretische Grundlagen, Konzeptionen, Prozesse und Effekte (S. 165-182). Münster: Waxmann.

Bauer, J., Drechsel, B., Retelsdorf, J., Sporer, T., Rösler, L., Prenzel, M., \& Möller, J. (2010). Panel zum Lehramtsstudium - PaLea: Entwicklungsverläufe zukünftiger Lehrkräfte im Kontext der Reform der Lehrerbildung. Beiträge zur Hochschulforschung, 32, 34-55.

Bauer, J., Diercks, U., Retelsdorf, J., Kauper, T., Zimmermann, F., Köller, O., Möller, J., \& Prenzel, M. (2011). Spannungsfeld Polyvalenz in der Lehrerbildung: Wie polyvalent sind Lehramtsstudiengänge und was bedeutet dies für die Berufswahlsicherheit der Studierenden? Zeitschrift für Erziehungswissenschaft, 14(4), 629-649.

Baumert, J., \& Kunter, M. (2006). Stichwort: Professionelle Kompetenz von Lehrkräften. Zeitschrift für Erziehungswissenschaft, 9(4), 469-520.

Baumert, J., \& Kunter, M. (2011). Das Kompetenzmodell von COACTIV. In M. Kunter, J. Baumert, W. Blum, U. Klusmann, S. Krauss \& M. Neubrand (Hrsg.), Professionelle Kompetenz von Lehrkräften. Ergebnisse des Forschungsprogramms COACTIV (S. 29-53). Münster: Waxmann.

Bennack, J., \& Jürgens, E. (2002). Schulpraktika in Lehramtsstudiengängen. In H.-U. Otto, T. Rauschenbach \& P. Vogel (Hrsg.), Erziehungswissenschaft: Lehre und Studium (S. 143-160). Opladen: Leske + Budrich.

Blanton, H., Buunk, B. P., Gibbons, F. X., \& Kuyper, H. (1999). When better-than-others compare upward: Choice of comparison and comparative evaluation as independent predictors of academic performance. Journal of Personality and Social Psychology, 76(3), 420-430.

Büscher, C. (2004). Schulpraktikum - ein Beitrag zur Professionalisierung in der Lehrerausbildung? Heidelberg: Universitätsbibliothek der Universität Heidelberg.

Chen, F.F. (2007). Sensitivity of goodness of fit indexes to lack of measurement invariance. Structural Equation Modeling, 14, 464-504.

Darge, K., Schreiber, M., König, J., \& Seifert, A. (2012). Lerngelegenheiten im erziehungswissenschaftlichen Studium. In J. König \& A. Seifert (Hrsg.), Lehramtsstudierende erwerben pädagogisches Professionswissen. Ergebnisse der Längsschnittstudie LEK zur Wirksamkeit der erziehungswissenschaftlichen Lehrerausbildung (S. 87-118). Münster: Waxmann.

Flagmeyer, D., \& Hoppe-Graff, S. (2006). Zu wenig Praxis, zu viel Theorie (Wissenschaft)? Ausgewählte Ergebnisse einer Befragung von Lehramtsstudierenden vor und nach den Schulpraktischen Studien. In M. Rotermund (Hrsg.), Schulpraktische Studien: Evaluationsergebnisse und neue Konzepte (S. 65-86). Leipzig: Leipziger Universitätsverlag.

Gröschner, A., \& Müller, K. (2014). Welche Rolle spielt die Dauer eines Praktikums? In K. Kleinespel (Hrsg.), Ein Praxissemester in der Lehrerbildung: Konzepte, Befunde und Entwicklungsperspektiven am Beispiel des Jenaer Modells (S. 62-75). Bad Heilbrunn: Klinkhardt.

Gröschner, A., Schmitt, C., \& Seidel, T. (2013). Veränderung subjektiver Kompetenzeinschätzungen von Lehramtsstudierenden im Verlauf eines Praxissemesters. Zeitschrift für Pädagogische Psychologie, 27(1-2), 77-86.

Gröschner, A., Müller, K., Bauer, J., Seidel, T., Prenzel, M., Kauper, T., \& Möller, J. (2015). Praxisphasen in der Lehrerausbildung - Eine Strukturanalyse am Beispiel des gymnasialen Lehramtsstudiums in Deutschland. Zeitschrift für Erziehungswissenschaft, 18(4), 639-665.

Hascher, T. (2006). Veränderungen im Praktikum - Veränderungen durch das Praktikum. Zeitschrift für Pädagogik, 51(Beiheft), 130-148.

Hascher, T. (2011). Forschung zur Wirksamkeit der Lehrerbildung. In E. Terhart, H. Bennewitz \& M. Rothlnad (Hrsg.), Handbuch der Forschung zum Lehrerberuf (S. 418-440). Münster: Waxmann.

Hascher, T. (2012). Lernfeld Praktikum - Evidenzbasierte Entwicklungen in der Lehrer/innenbildung. Zeitschrift für Bildungsforschung, 2, 109-129.

Hascher, T., \& Moser, P. (1999). Lemen im Praktikum - die Rolle der Praktikumsleitenden in berufspraktischen Ausbildung. Bildungsforschung und Bildungspraktis, 21(3), 312-355.

Hascher, T., \& Moser, P. (2001). Betreute Praktika - Anforderungen an Praktikumslehrerinnen und -lehrer. Beiträge zur Lehrerbildung, 19(2), 217-231. 
Hascher, T., \& de Zordo, L. (2015). Langformen von Praktika. Ein Blick auf Österreich und die Schweiz. Journal für Lehrerinnen- und Lehrerbildung, 15(1), 22-32.

Herzberg, P. Y., \& Brähler, E. (2006). Assessing the big-five personality domains via short forms. A cautionary note and a proposal. European Journal of Psychological Assessment, 22(3), 139-148.

Holtz, P. (2014). Es heißt ja auch Praxissemester und nicht Theoriesemester: Quantitative und qualitative Befunde zum Spannungsfeld zwischen „Theorie“ und „Praxis“ im Jenaer Praxissemester. In K. Kleinespel (Hrsg.), Ein Praxissemester in der Lehrerbildung. Konzepte, Befunde und Entwicklungssperspektiven am Beispiel des Jenaer Modell (S. 97-118). Bad Heilbrunn: Klinkhardt.

Hox, J. J. (2002). Multilevel analysis: Techniques and applications. Mahwah: Lawrence Erlbaum.

Kleinke, K., Schlüter, E., \& Christ, O. (2017). Strukturgleichungsmodelle mit Mplus. Eine praktische Einführung. Berlin: De Gruyter.

Klostermann, M., Höffler, T.N., Bernholt, A., Busker, M., \& Parchmann, I. (2014). Erfassung und Charakterisierung kognitiver und affektiver Merkmale von Studienanfängern im Fach Chemie. Zeitschrift für Didaktik der Naturwissenschaften, 20(1), 101-113.

KMK (2005). Eckpunkte für die gegenseitige Anerkennung von Bachelor- und Masterabschlüssen in Studiengängen, mit denen die Bildungsvoraussetzungen für ein Lehramt vermittelt werden (Beschluss der KMK vom 02.06.2005; sog. Quedlinburger Beschluss). https://www.kmk.org/fileadmin/ veroeffentlichungen_beschluesse/2005/2005_06_02-Bachelor-Master-Lehramt.pdf

König, J. (2012). Zum Einfluss der Schulpraxis im Lehramtsstudium auf den Erwerb von pädagogischem Wissen: Spielen erste Unterrichtsversuche eine Rolle? In T. Hascher \& G. H. Neuweg (Hrsg.), Forschung zur (Wirksamkeit der) Lehrer/innen/bildung. Wien: Lit Verlag.

König, J., Tachtsoglou, S., \& Seifert, A. (2012). Individuelle Voraussetzungen, Lerngelegenheiten und der Erwerb von pädagogischen Professionswissen. In J. König \& A. Seifert (Hrsg.), Lehramtsstudierende erwerben pädagogisches Professionswissen. Münster: Waxmann. ISBN 978-3830926252.

Lazarides, R., \& Mohr, S. (2015). Lehramtsstudierende im Orientierungspraktikum: Wie wird die universitäre Praktikumsbetreuung wahrgenommen? Beiträge zur Lehrerinnen- und Lehrerbildung, 33(3), 380-392. https://www.pedocs.de/volltexte/2017/13910/pdf/BZL_2015_3_380_392.pdf.

Lipowsky, F. (2003). Wege von der Hochschule in den Beruf - Eine empirische Studie zum beruflichen Erfolg von Lehramtsabsolventen in der Berufseinstiegsphase. Bad Heilbrunn: Klinkhardt.

Malmberg, L.-E. (2008). Student teachers' achievement goal orientations during teacher studies: Antecedents, correlates and outcomes. Learning and Instruction, 18(5), 438-452.

Marsh, H.W., \& Martin, A.J. (2011). Academic self-concept and academic achievement: Relations and causal ordering. British Journal of Educational Psychology, 81, 59-77.

Möller, J., \& Marsh, H.W. (2013). Dimensional comparison theory. Psychological Review, 120(3), 544-560.

Möller, J., \& Trautwein, U. (2009). Selbstkonzept. In E. Wild \& J. Möller (Hrsg.), Pädagogische Psychologie (S. 179-204). Berlin: Springer.

Müller, K. (2010). Das Praxisjahr in der Lehrerbildung. Empirische Befunde zur Wirksamkeit studienintegrierter Langzeitpraktika. Bad Heilbrunn: Julius Klinkhardt.

Muthén, B., \& Muthén, L. (2013). Mplus (Version 7.11). Los Angeles: StatModel.

Reinhoffer, B., \& Dörr, G. (2008). Zur Wirksamkeit Schulpraktischer Studien. In M. Rotermund (Hrsg.), Bologna verändert die Lehrerbildung. Auswirkungen der Hochschulreform (S. 10-31). Leipzig: Leipziger Universitätsverlag.

Retelsdorf, J., Bauer, J., Gebauer, S. K., Kauper, T., \& Möller, J. (2014). Erfassung berufsbezogener Selbstkonzepte von angehenden Lehrkräften (ERBSE-L). Diagnostica, 60(2), 98-110. https://doi.org/10. 1026/0012-1924/a000108.

Rösler, L., Zimmermann, F., Bauer, J., Möller, J., \& Köller, O. (2013). Interessieren sich Lehramtsstudierende für bildungswissenschaftliche Studieninhalte? Eine Längsschnittstudie vom ersten bis zum vierten Semester. Zeitschrift für Pädagogik, 59(1), 24-42.

Rothland, M., \& Boecker, S. K. (2014). Wider das Imitationslernen in verlängerten Praxisphasen. Potenzial und Bedingungen des Forschenden Lernens im Praxissemester. Die Deutsche Schule, 106(4), 389-400.

Rothland, M., \& Boecker, S. K. (2015). Viel hilft viel? Forschungsbefunde und -perspektiven zum Praxissemester in der Lehrerbildung. Lehrerbildung auf dem Prüfstand, 8(2), 112-134.

Rothland, M., \& Straub, S. (2018). Die Veränderung berufsbezogener Selbstkonzepte im Praxissemester. Empirische Befunde zur Bedeutung sozialer Unterstützung durch betreuende Lehrkräfte sowie Kommilitoninnen und Kommilitonen. In J. König, M. Rothland \& N. Schaper (Hrsg.), Learning to Practice, Learning to Reflect? Ergebnisse aus der Längsschnittstudie LtP zur Nutzung und Wirkung des Praxissemesters in der Lehrerbildung (S. 135-162). Wiesbaden: Springer VS. 
Schafer, J.L., \& Graham, J. W. (2002). Missing data: Our view of the state of the art. Psychological Methods, 7, 147-177.

Scheller, R., \& Filipp, S.-H. (1995). Selbstkonzept - Berufskonzept. In W. Sarges (Hrsg.), ManagementDiagnostik (S. 288-295). Göttingen: Hogrefe.

Schubarth, W., Gottmann, C., \& Krohn, M. (2014). Wahrgenommene Kompetenzentwicklung im Praxissemester und dessen berufsorientierende Wirkung: Ergebnisse der ProPrax-Studie. In K.-H. Arnold, A. Gröschner \& T. Hascher (Hrsg.), Schulpraktika in der Lehrerbildung. Münster: Waxmann.

Schubarth, W., Speck, K., \& Seidel, A. (Hrsg.). (2011). Nach Bologna: Praktika im Studium - Pflicht oder Kür? Empirische Analysen und Empfehlungen für die Hochschulpraxis. Potsdam: Universitätsverlag Potsdam.

Schüle, C., Besa, K.-S., Schriek, J., \& Arnold, K.-H. (2017). Die Veränderung der Lehrerselbstwirksamkeitsüberzeugung in Schulpraktika. Zeitschrift für Bildungsforschung, 7, 23-40.

Schwippert, K., Feld, I., Doll, J., \& Buchholtz, N. (2013). Vergleich motivationaler und volitionaler Bedingungen des selbst eingeschätzten Studienerfolgs von Lehramtsstudierenden in zwei Studienabschnitten. In S. Blömeke, A. Bremerich-Vos, G. Kaiser, G. Nold, H. Haudeck, J.-U. Keßler \& K. Schwippert (Hrsg.), Professionelle Kompetenzen im Studienverlauf. Weitere Ergebnisse zur Deutsch-, Englischund Mathematiklehrerausbildung aus TEDS-LT (S. 231-249). Münster: Waxmann.

Seifert, A., \& Schaper, N. (2018). Die Veränderung von Selbstwirksamkeitserwartungen und der Berufswahlsicherheit im Praxissemester. In J. König, M. Rothland \& N. Schaper (Hrsg.), Learning to Practice, Learning to Reflect? Ergebnisse aus der Längsschnittstudie LtP zur Nutzung und Wirkung des Praxissemesters in der Lehrerbildung (S. 135-162). Wiesbaden: Springer VS.

Terhart, E. (Hrsg.). (2000). Perspektiven der Lehrerbildung in Deutschland. Abschlussbericht der von der Kultusministerkonferenz eingesetzten Kommission. Weinheim: Beltz.

Terhart, E. (2002). Standards für die Lehrerbildung. Eine Expertise für die Kultusministerkonferenz. Münster: Institut für Schulpädagogik und allgemeine Didaktik. Westfälische Wilhelms-Universität Münster.

Topsch, W. (2004). Schulpraxis in der Lehrerbildung. In S. Blömeke, P. Reinhold, G. Tulodziecki \& J. Wildt (Hrsg.), Handbuch Lehrerbildung (S. 476-486). Bad Heilbrunn: Klinkhardt.

van der Veen, I., de Jong, U., van Leeuwen, M., \& Korteweg, J. A. (2005). The development of higher education students' interest in th eir subject: The case of higher professional education in the Netherlands. Studies in Higher Education, 30(3), 275-289.

Weyland, U. (2012). Expertise zu den Praxisphasen in der Lehrerbildung in den Bundesländern. Hamburg: Landesinstitut für Lehrerbildung und Schulentwicklung. 